\title{
Retina-Driven Dephosphorylation of the NR2A Subunit Correlates with Faster NMDA Receptor Kinetics at Developing Retinocollicular Synapses
}

\author{
Matthew Townsend, ${ }^{1}$ Yudong Liu, ${ }^{2}$ and Martha Constantine-Paton ${ }^{3}$ \\ ${ }^{1}$ Interdepartmental Neuroscience Program, Yale University, New Haven, Connecticut 06520, ${ }^{2}$ Department of Biochemistry and Biophysics, The University \\ of North Carolina, Chapel Hill, North Carolina 27599, and ${ }^{3}$ Departments of Biology and Brain and Cognitive Science and the McGovern Institute for Brain \\ Research, Massachusetts Institute of Technology, Cambridge, Massachusetts 02139
}

We describe a homeostatic mechanism that limits NMDA receptor currents in response to early light activation of a developing visual pathway. During the second postnatal week of rodent retinocollicular development, the $\mathrm{Ca}^{2+}$-activated phosphatase calcineurin (CaN) mediates a rapid, activity-induced shortening in the decay time of NMDA receptor (NMDAR) currents. We show that protein kinase A acts in opposition to CaN to maintain NMDAR currents with long decay times. The CaN-mediated change is coincident with the initial expression of the NMDAR subunit NR2A. Using NR2A knock-out mice and dialyzing neurons with a constitutively active CaN, we demonstrate that NR2A subunits are necessary for the effect of CaN on NMDAR current kinetics. In wild-type mice, Ser900 of NR2A, previously implicated in $\mathrm{CaN}$-mediated glycine-independent desensitization, becomes chronically dephosphorylated by postnatal day 11 as NMDAR current decay times become faster. Pharmacologically disrupting early photoreceptor-driven activity in the retina eliminates the dephosphorylation of NR2A and prevents the shortening in NMDAR current decay time. These data suggest that the developmental onset of retinal activity increases $\mathrm{CaN}$-mediated dephosphorylation of NR2A subunits newly incorporated into synaptic NMDARs of the superior colliculus, thereby providing a mechanism for the early and rapid reduction of NMDAR current decay time in visual neurons.

Key words: NMDA; calcineurin; superior colliculus; development; visual; plasticity

\section{Introduction}

The NMDA-type glutamate receptor (NMDAR) is a tetrameric, integral membrane protein consisting of two obligatory NR1 subunits and two NR2 subunits (A-D) (Rosenmund et al., 1998). The composition of NMDARs is developmentally regulated, such that NR2B subunits are highly expressed in the forebrain and midbrain of juveniles, whereas NR2A-containing receptors predominate in adulthood (Watanabe et al., 1992; Monyer et al., 1994). The kinetics of the NMDAR current decay time can be modified by several factors including subunit composition (Vicini et al., 1998). Sensory deprivation experiments, which delay the close of the critical period in visual cortex (Lindlbauer et al., 1998; Nase et al., 1999), also postpone the shortening of the NMDAR current decay time and the addition of NR2A to synapses (Carmignoto and Vicini, 1992; Quinlan et al., 1999).

NMDAR function can be regulated by phosphorylation (Ali and Salter, 2001), in addition to subunit composition. Many reports have concluded that the activation of kinases increases the NMDAR current by favoring the open state of the channel (Wang

\footnotetext{
Received March 31, 2004; revised 0ct. 25, 2004; accepted 0ct. 30, 2004.

This work was supported by National Institutes of Health Grant NS32290/EY006039 to M.C.-P., by National Institute of Mental Health Grant 5T32DA07290 to M.T., and by National Eye Institute Grant 5T32EY07115 to M.T.

Correspondence should be addressed to Martha Constantine-Paton, Department of Biology, Massachusetts Institute of Technology, Room 68-380, 77 Massachusetts Avenue, Cambridge, MA 02139-4301. E-mail: mcpaton@mit.edu.

DOI:10.1523/JNEUROSCI.1207-04.2004

Copyright $\odot 2004$ Society for Neuroscience $\quad$ 0270-6474/04/2411098-10\$15.00/0
}

et al., 1994b; Raman et al., 1996; Rostas et al., 1996). In contrast, the $\mathrm{Ca}^{2+} /$ calmodulin-dependent Ser/Thr phosphatase calcineurin $(\mathrm{CaN})$ reduces the open time and open probability of NMDARs (Lieberman and Mody, 1994) and desensitizes the receptor in hippocampal neurons (Tong et al., 1995).

$\mathrm{CaN}$ is also important in synaptic plasticity. CaN is essential for depotentiation after the induction of long-term potentiation (LTP) (Zhuo et al., 1999; Zeng et al., 2001). Transgenic mice with reduced $\mathrm{CaN}$ function show enhanced LTP and improved performance in memory tasks (Malleret et al., 2001). CaN is localized to the postsynaptic density complex through the scaffolding molecule A-kinase associated protein 79/150 (Colledge et al., 2000) and is therefore positioned to rapidly respond to changes in synaptic $\mathrm{Ca}^{2+}$. Thus, $\mathrm{CaN}$ is implicated in a number of postsynaptic signaling events.

We have shown previously in the superior colliculus (SC) of rats that $\mathrm{CaN}$ is activated by $\mathrm{Ca}^{2+}$ influx through NMDARs near the end of the second postnatal week (Shi et al., 2000). Once activated, $\mathrm{CaN}$ induces a persistent shortening of the decay time of synaptic NMDAR currents. We now report that mice undergo a similar developmental shortening of NMDAR current decay time by postnatal day 11 (P11), as in the rat. Extending our previous findings, we show that reduction of early retinal activity in P6 mice prevents this shortening by P11. Using constitutively active $\mathrm{CaN}$ peptides in the patch pipette and NR2A knock-out (NR2AKO) mice, we demonstrate that the presence of the NR2A subunit in the receptor is necessary for the CaN-mediated effect 
on current decay time. Moreover, dephosphorylation of Ser900 of the NR2A subunit is tightly correlated with the CaN-mediated changes in NMDAR current decay time. This change in NMDAR function may play an important role in developmental plasticity in the retinocollicular pathway, because it is coincident with the final NMDAR-dependent structural refinement of the retinocollicular map (Simon et al., 1992; McLaughlin et al., 2003). It may also constitute a novel homeostatic mechanism that can rapidly control NMDAR $\mathrm{Ca}^{2+}$ flux in response to early changes in the level and pattern of input activity.

\section{Materials and Methods}

Animals. NR2A knock-out mice were produced in the laboratory of M. Mishina (University of Tokyo, Tokyo, Japan) and kept as homozygotes as assessed by PCR analysis of tail DNA. All surgical procedures were compliant with the Massachusetts Institute of Technology Institutional Animal Care and Use Committee animal protocol review.

Surgery. Elvax plastic (DuPont, Billerica, MA) was prepared as in the studies by Simon et al. (1992) and Townsend et al. (2003). Briefly, the Elvax polymer was dissolved in dichloromethane and vortexed with glutamate receptor antagonists such that the final antagonist concentration was $6 \mathrm{~mm}(+)$-5-methyl-10,11-dihydro-5H-dibenzo [a,d] cyclohepten5,10-imine maleate (MK-801) and $600 \mu \mathrm{M}$ 2,3-dihydroxy-6-nitro-7sulfonyl-benzo[f] quinoxaline-7-sulfonamide (NBQX) (antag/Elvax). For sham treatment, the Elvax contained DMSO alone (sham/Elvax). Cryostat sections of Elvax (300 $\mu \mathrm{m}$ thick) were cut into slivers $(\sim 1.25$ $\mathrm{mm}$ long) and sterilized before being implanted in the eye (Prusky and Ramoa, 1999). Surgery was accomplished under halothane anesthesia on P6. After opening the eyelid, a small incision was made at the ciliary margin of the eye, and a sliver of Elvax was slipped into the vitreous humor. The eyelid was sutured shut and lightly glued with Vetbond. Pups received a single injection of buprenorphine after the surgery as an analgesic. The Elvax volume was approximately one-thirtieth of the volume of the eye. Thus, the antag/Elvax slivers delivered an initial active concentration of $\sim 200 \mu \mathrm{M}$ MK-801 and $\sim 20 \mu \mathrm{M}$ NBQX.

To assess the effectiveness of the visual impairment with Elvax, we tested for a pupillary reflex in the operated eye (Prusky and Ramoa, 1999). On P11, the eyelids were sequentially opened starting with the operated eye. The sclera was inspected for proper healing after surgery. A hand-held penlight or a fiber optic light attached to a dissecting scope was flashed into one eye. The iris was visually inspected for the occurrence of a pupillary reflex. Animals receiving antag/Elvax typically showed little to no response to the light, whereas the contralateral eye or a sham/Elvax eye showed evident constriction. In no instance was there evidence of cataract occlusion of the pupil. After deeply anesthetizing the mice, they were decapitated, and both the superior colliculus and the treated eye were removed. The eye was then dissected to confirm the correct positioning of the Elvax in the eye. It is difficult to ascertain precise placement of the Elvax in vivo, because the dissection of the eye could easily cause the Elvax to move. Furthermore, because the Elvax is transparent, it cannot be clearly observed through the pupil. In two cases, we observed a pupillary response in "operated" eyes, and determined afterward that the Elvax was exterior to the eye cup, because of either misplacement or ejection through the point incision after suturing the eyelid. These animals were excluded from the study. A schematic representation of this procedure is shown in supplemental Figure A (available at www.jneurosci.org as supplemental material).

Western blotting. Synaptosomes were prepared from P9 and P11 mice according to previously published methods (Dunah and Standaert, 2001) with minor changes. Briefly, SCs from one litter of mice were dissected and homogenized in $4^{\circ} \mathrm{C}$ TEVP buffer (10 mM Tris- $\mathrm{HCl}, 1 \mathrm{~mm}$ EDTA, 1 mм EGTA, pH 7.4, $320 \mathrm{~mm}$ sucrose, complete protease inhibitors) (Roche, Indianapolis, IN). Samples were spun at $1000 \times g$ at $4^{\circ} \mathrm{C}$ for 5 $\min$ to remove nuclei. The supernatant was then spun at $10,000 \times g$ for 5 min to pellet insoluble homogenate. The pellets were lysed with a hypoosmotic buffer (TEVP without sucrose) with $0.2 \%$ Triton X-100 and centrifuged at $25,000 \times g$ to obtain a synaptosomal fraction. The pellet was resuspended in Laemli buffer and stored at $-80^{\circ} \mathrm{C}$. Protein levels were quantified in a spectrophotometer. See supplemental material B (available at www.jneurosci.org) for characterization of the enrichment.

Samples were run on one-dimensional 6 or $8 \%$ SDS-PAGE gels and transferred to polyvinylidene difluoride membranes for Western blotting. Blots were probed with antibodies to CaN (Sigma, St. Louis, MO; BD Transduction, San Jose, CA), actin (Sigma), and NR2A (Molecular Probes, Eugene, OR) and reacted for chemiluminescence (Pierce, Rockford, IL). Film exposures were selected to be in the linear range for all bands being compared. Actin bands on the same blot were used to corroborate equal loading. Band density was quantified as described previously using NIH Image and its gel-plotting macros (Shi et al., 1997). At least three separate protein isolations (three litters) were used at all time points and averaged for quantification. For normalization of phosphoSer900 (P-Ser900) in Figure 5, the optical density (OD) of a given phospho-Ser900 band was divided by the average for the blot. The membrane was then stripped and reprobed with an anti-NR2A antibody. The OD of a given NR2A band was then divided by the average for the blot. The first number was then divided by the second to give the normalized OD [(example, phospho-day 9/avg phospho-day 9, 11, 15)/(NR2A-day 9/avg NR2A-day 9, 11, 15)].

Electrophysiology. Recordings were made from neurons of the stratum griseum superficiale of $350 \mu \mathrm{m}$ parasagittal superior colliculus slices at room temperature. Slices were equilibrated in artificial CSF consisting (in mM): $117 \mathrm{NaCl}, 3 \mathrm{MgCl}_{2}, 4 \mathrm{KCl}, 3 \mathrm{CaCl}_{2}, 1.2 \mathrm{NaHPO}_{4}, 26 \mathrm{NaHCO}_{3}$, 0.002 glycine, and 16 glucose for at least $1 \mathrm{hr}$ before recording. To facilitate rapid solution exchange, the recording chamber used was designed to perfuse slices from both sides. Borosilicate glass pipettes were filled with the following (in mM): 122.5 Cs-gluconate, $17.5 \mathrm{CsCl}, 10 \mathrm{HEPES}$ (CsOH), 0.2 Na-EGTA, 2 Mg-ATP, 0.3 Na-GTP, and $8 \mathrm{NaCl}$, pH 7.3. Cells had resting potentials of approximately $-50 \mathrm{mV}$ and were voltage clamped at $-70 \mathrm{mV}$. NMDAR currents were pharmacologically isolated at $-70 \mathrm{mV}$ in $0 \mathrm{mM} \mathrm{Mg}^{2+}$ by the addition of a $20 \mu \mathrm{M}$ concentration of the $\mathrm{GABA}_{\mathrm{A}}$ receptor antagonist picrotoxin and a $20 \mu \mathrm{M}$ concentration of the AMPA/kainate receptor (AMPA/KAR) antagonist (NBQX, DNQX, or CNQX; Tocris Cookson, Ellisville, MO). CaN was inhibited with $1 \mu \mathrm{M}$ FK506/Tacrolimus [Calbiochem (La Jolla, CA), but now patented by Fujisawa Pharmaceutical (Osaka, Japan)] and protein phosphatase 1 and 2A (PP1/PP2A) by $1 \mu \mathrm{M}$ okadaic acid (Tocris Cookson). In some experiments, the following inhibitors and activators of kinases and phosphatases were used: $5 \mu \mathrm{M}$ protein kinase A (PKA)-inhibitory peptide and 5 $\mu \mathrm{M}$ protein kinase $\mathrm{C}$ ( $\mathrm{PKC}$ )-inhibitory peptide (Upstate Biotechnology, Charlottesville, VA); calcium calmodulin-dependent protein kinase II (CaMKII) inhibitors $(10 \mu \mathrm{M}) \mathrm{KN}-62$ or KN-93, PKA activator $(20 \mu \mathrm{M})$ 8-Br-cAMP, and PKC antagonist GK-10923X (Tocris Cookson). Constitutively active CaN was prepared by Yudong Liu (The University of North Carolina, Chapel Hill, NC) in the laboratory of Hengming Ke (The University of North Carolina) in collaboration with Jun Liu (Johns Hopkins University, Baltimore, MD) using a baculovirus expression system as described by Perrino et al. (1992). Briefly, the CaNA $\alpha$ subunit was expressed in Sf9 cells. The protein was purified by calmodulin-Sepharose affinity chromatography and diluted to a concentration of $1 \mu \mathrm{M}$ in intracellular recording pipette solution. All neurons maintained seal resistances between 1 and $3 \mathrm{G} \Omega$, and access resistance was $\leq 40 \mathrm{M} \Omega$. Pipette and whole-cell capacitance were compensated.

Average spontaneous synaptic currents were collected within a 2-8 min period after a solution exchange period of $5 \mathrm{~min}$. Spontaneous events were considered synaptic currents if they had a rise time $<8 \mathrm{msec}$ and peak amplitudes at least two times baseline-peak noise (1.5 pA). Synaptic currents were averaged using MiniAnalysis software (SynaptoSoft, Decatur, GA). Averages were derived from at least 15 single events and fit with a single exponential to estimate decay time (90-37\% peak amplitude) ([see supplemental material C for sample curve fits (available at www.jneurosci.org)]. This accounts for the slight difference in kinetics reported in this publication compared with our previous work, where decay time was estimated as the first point of intersection at $37 \%$ with the average spontaneous trace (Shi et al., 2000). In that previous publication, we also reported on the decay time of individual spontaneous NMDAR currents in addition to the average. We showed that both the individual and average NMDAR current decay times shorten simultaneously by P11. 
To induce the CaN-mediated shortening of NMDAR currents in P9 and P10 slices (see Fig. 3), a 2-10 $\mu \mathrm{A}$ current was delivered through tungsten or platinum iridium bipolar electrode placed in the stratum opticum of SC slices. The stimulation protocol consisted of four pulses delivered at $100 \mathrm{~Hz}$ every second for $45 \mathrm{~min}$ as in the study by Shi et al. (2000).

Statistics. Statistics were performed using NCSS 2000 software (NCSS Statistical Software, Kaysville, UT). Statistical significance was determined using Tukey-Kramer post hoc test for all ANOVAs. Error bars indicate the SEM.

\section{Results}

Whole-cell voltage-clamp recordings were made from sagittal brain slices of the superficial visual layers of the SC (sSC) of mice between the ages of P7 and P15. NMDAR currents were isolated by holding neurons at $-70 \mathrm{mV}$ in $0 \mathrm{~mm} \mathrm{Mg}^{2+}$ while pharmacologically blocking AMPA/KA and $\mathrm{GABA}_{\mathrm{A}}$ receptors. Addition of the NMDAR antagonist D-AP-5 eliminated all detectable responses as in the study by Shi et al. (2000).

\section{CaN mediates a rapid shortening of the}

\section{NMDAR current decay time in wild-type mice}

In rats, CaN mediates a rapid shortening of the NMDAR current decay time overnight between P10 and P11 (Shi et al., 2000). Mice demonstrate a similar change in the NMDAR current decay time (Fig. $1 A$ ) beginning on P10 and completed by P11. Figure $1 B$ shows three sample traces of the average spontaneous NMDAR current recorded from individual neurons on P8 and P11. Addition of the membrane-permeable CaN antagonist FK506 (1 $\mu \mathrm{M})$ to the perfusion media on P8 had no effect on the long decay time of P8 NMDAR currents (data not shown) (Shi et al., 2000). However, on P11, perfusion with FK506 caused a shift in the kinetics of the NMDAR current to a long decay time similar to P8. As in the rat, the shortening in decay time between P8 and P11 occurred without a significant change in current amplitude. The mouse NMDAR current decay time was $53 \pm 6.15 \%(p<0.05)$ faster within $24-36 \mathrm{hr}$, a rate fully comparable with that observed previously in rats.

Results similar to those obtained with FK506 were also observed when cyclosporin was used to block CaN (data not shown). However, to address the possibility that CaN may regulate the NMDAR current indirectly through the phosphatases PP1 and PP2A (Wang et al., 1994a), we tested the effect of the PP1/2A antagonist okadaic acid $(1 \mu \mathrm{M})$ on NMDAR current decay time. As shown in Figure $1 C$, bath application of okadaic acid failed to prolong the NMDAR decay time at this age $(p>0.05)$. These data suggest that in the sSC, as in the hippocampus (Lieberman and Mody, 1994), CaN can act directly on NMDARs.

\section{Retinal ganglion cell activity is necessary for the CaN-induced shortening of NMDAR currents} Because the effect of CaN on NMDAR current kinetics consistently occurred on P10-P11, we sought to identify the rele-
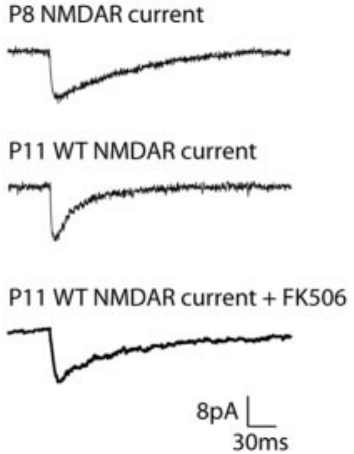

P11 WT NMDAR current
C

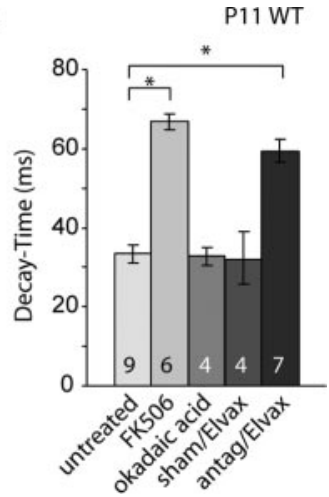

Figure 1. CaN mediates a rapid shortening of spontaneous NMDAR current decay time in sSC neurons during development. $A$, The decay time ( $90-37 \%$ peak amplitude) of NMDAR currents becomes significantly shorter between P9 and P11 (Student's t test;

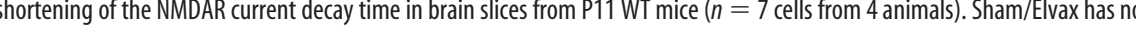
different from untreated (ANOVA Tukey-Kramer post hoc; ${ }^{*} p<0.01$ ). Numerals in histogram bars in this and following figures indicate the number of cells per experimental condition. Error bars indicate SEM.

vant in vivo mechanism responsible for regulating the decay time of NMDAR currents. Because stimulating the stratum opticum in P9 rat slices can precociously activate $\mathrm{CaN}$, we predicted that the in vivo mechanism was likely to involve altered synaptic activity at P10-P11. The biological mechanism had to be distinct from eye opening, which does not occur until P13. Several reports have suggested that the maturation of the photoreceptor-bipolarganglion cell pathway occurs close to P10 (Miller et al., 1999) and that glutamate receptors begin to play a prominent role in driving retinal activity by P11 (Bansal et al., 2000). To test whether this maturation of the glutamatergic pathway in the retina was necessary for the activation of $\mathrm{CaN}$ in the $\mathrm{SSC}$, we reduced retinal glutamatergic activity in vivo and assayed the effects on NMDAR currents in sSC neurons. Slivers of Elvax plastic infused with glutamate receptor antagonists NBQX and MK-801 were placed in one eye of P6 mice (Prusky and Ramoa, 1999; Townsend et al., 2003). This method was preferable to dark rearing, because both evoked and spontaneous glutamatergic activity could be reduced. Furthermore, the contralateral eye served as a control for the operated eye. The slow release of these glutamate receptor antagonists reduced the retinal response in the treated eye several days later as determined by the absence of a pupillary constriction to flashes of light.

Figure 2. PKA lengthens the spontaneous NMDAR current decay time in opposition to CaN. A, Schematic model for regulation of NMDAR phosphorylation by CaN and a kinase. In the left panel, the kinase activity exceeds phosphatase activity resulting in a phosphorylated receptor and long NMDAR current decay times. In the middle panel, CaN activity exceeds kinase activity resulting in a dephosphorylated receptor and short NMDAR current decay times. The right panel depicts the model experiment shown in $B$ and C. By first inhibiting the correct kinase, CaN should dephosphorylate the receptor causing a shortened NMDAR current. By then inhibiting CaN in the same experiment, the current should remain short, because the critical kinase cannot rephosphorylate the receptor. However, if the incorrect kinase is inhibited, then blocking CaN activity should allow for the rephosphorylation of the receptor restoring slow NMDAR currents as in the left panel. B, PKA inhibitory peptide was applied through the patch pipette 20 min before adding the CaN antagonist FK506. Preinhibition of PKA prevents the lengthening of NMDAR current decay times after addition of FK506 (top two traces; Student's $t$ test, $p=0.16$, comparing before and after FK506). In contrast, PKC inhibitors (second row, traces), applied postsynaptically via the patch pipette (PKC-inhibitory peptide) ( $A, B$, gray bar) (Figure legend continues) 
A

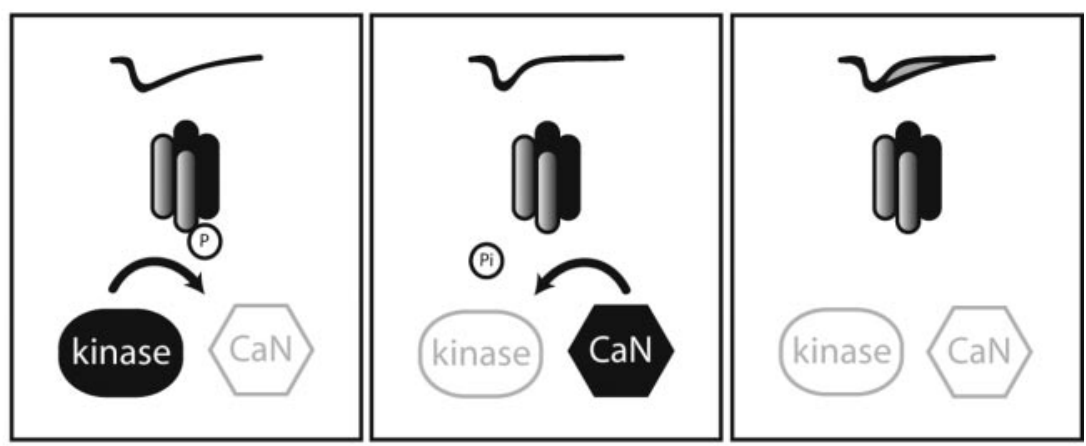

B

Before FK506

After FK506

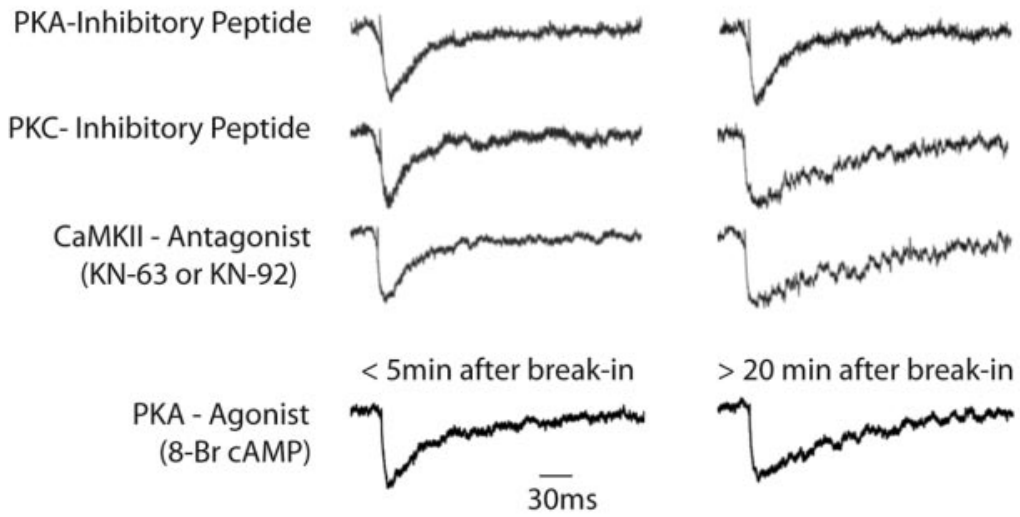

C

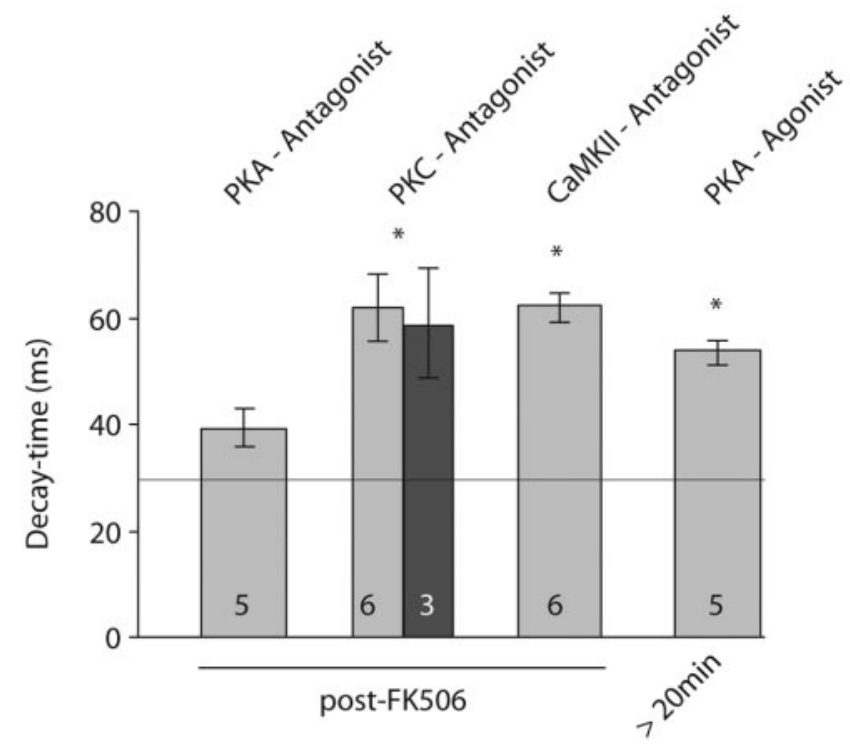

(Figure legend continued) or bath applied (GK10923X) ( $B$, black bar), did not prevent the lengthening of NMDAR current decay time by FK506 (Student's $t$ test; $p<0.01$ and $p<0.05$, respectively, comparing before and after FK506). CaMKII antagonists (KN-62 or KN-93; third row, traces) also did not abolish the shift in NMDAR current decay time in the presence of FK506 (Student's $t$ test; $p<0.01$, comparing before and after FK506). Addition of the membrane-permeable PKA agonist 8-Br-cAMP to P11 neurons (bottom row, traces) overcame the effect of endogenous CaN and prolonged the decay time of NMDAR current (Student's $t$ test; ${ }^{*} p<0.01$ ). All neurons were recorded in P11 slices. C, Histogram illustrating the effect of various agents that antagonize or facilitate specific kinases on the decay time of NMDAR currents in P11 neurons. The line through the histograms represents the average normal NMDAR decay time at P11.
We tested whether disrupting photoreceptor-driven activity in the eye from $\mathrm{P} 6$ would affect the decay time of the NMDAR current in the SSC neurons on P11. Spontaneous NMDAR currents were recorded in P11 collicular slices either ipsilateral or contralateral to the antag/ Elvax-treated eye. As shown in Figure 1C, antag/Elvax prevented the shorting of NMDAR current decay time normally seen by P11. In contrast, NMDAR currents recorded from ipsilateral collicular neurons of the same mice showed fast decaying NMDAR currents that are typical on P11, thus indicating that the glutamate receptor antagonists were restricted to one eye. Fast decaying NMDAR currents were also present in P11 littermates with sham/ Elvax in the vitreous of the contralateral eye. Thus, glutamatergic activity in the retina is necessary for the shortening of NMDAR current decay times in the sSC of mice on P10-P11.

\section{PKA opposes CaN-induced shortening in NMDAR current decay time}

We sought to identify which neuronal kinase(s) balance the CaN-mediated shortening of NMDAR current decay time. The results from Figure 1 suggested that $\mathrm{CaN}$ antagonists induce a lengthening of NMDAR current decay time in P11 neurons by allowing for the rephosphorylation of CaN substrates. Therefore, we reasoned that blocking the appropriate kinase before adding FK506 should prevent this rephosphorylation, and NMDAR currents would remain fast. In contrast, blockade of an inappropriate kinase should allow for rephosphorylation and lengthening of the NMDAR current decay time after perfusion with FK506 (Fig. 2A). To this end, antagonists for PKA or PKC or CaMKII were dialyzed into P11 sSC neurons through the whole-cell pipette or added to the perfusion media, and we determined which antagonists caused NMDAR currents to remain fast even after FK506 was added to the perfusion.

Figure $2 B$ (top two traces) shows the average NMDAR current from a single neuron in which the PKA-inhibitory peptide was introduced into the recording pipette solution (Wang et al., 1994b). After break-in, the cell was dialyzed for $20 \mathrm{~min}$, and the decay times of spontaneous NMDAR currents were recorded. NMDAR current kinetics was fast, which is typical of other P11 neurons. However, when FK506 was added to the perfusion media, the PKA-inhibitory peptide prevented the lengthening of the NMDAR current decay time that normally occurred with $\mathrm{CaN}$ antagonists. These results suggest 
that inhibition of PKA prevents the rephosphorylation of the critical CaN sites, which are necessary for causing long NMDAR currents. Thus, active PKA is predicted to be associated with long NMDAR currents. This finding is consistent with previous work suggesting that PKA balances CaN-mediated desensitization of NMDARs in hippocampal neurons (Raman et al., 1996).

We also tested PKC and CaMKII inhibitors using the same protocol. Neither PKC nor CaMKII antagonists prevented the lengthening of the NMDAR current decay time by FK506 (Fig. 2 B, middle four traces). Thus, PKC and CaMKII do not oppose $\mathrm{CaN}$ in regulating NMDAR current decay time at this age.

If PKA activity acts alone to counter the effects of CaN on the NMDAR current, we reasoned that overactivation of PKA in P11 neurons should lengthen the NMDAR current decay time despite endogenous CaN activity. NMDAR current decay times were compared before and after adding the nonhydrolyzable cAMP analog 8-Br cAMP to the pipette solution and/or perfusion (Fig. $2 \mathrm{~B}$, bottom two traces). With this procedure, NMDAR current kinetics was significantly longer when measured after $20 \mathrm{~min}$. These results are summarized in Figure 2C. Therefore, PKA activity appears to be both necessary and sufficient to reverse the tonic effect of $\mathrm{CaN}$ on NMDAR currents in the sSC.

\section{NR2AKO mice lack stimulation-induced shortening in NMDAR current decay time}

We have shown previously that synaptic NMDAR currents decrease in amplitude in the sSC of NR2AKO mice beginning on P9, suggesting that the first NR2A-containing receptors are gradually inserted into the synapses of Wild-type (WT) visual neurons starting at this age (Townsend et al., 2003). Therefore, we used NR2AKO mice to further our understanding of the interaction between NR2A and CaN in regulating NMDAR current decay time.

Each square in Figure $3 A$ represents the average decay time of NMDAR currents recorded from an NR2AKO sSC neuron. Unlike WT animals, the NMDAR current decay times remain long in NR2AKO neurons even by P11. Thus, the absence of NR2A prevented the developmental shortening of NMDAR currents. However, NMDAR currents are difficult to detect by P11 in these mice because of the loss of NR2A-containing receptors (Townsend et al., 2003). Therefore, one explanation for the persistence of long NMDAR currents in mutant mice is that the decrease in NMDAR current amplitude in NR2AKO mice could prevent a sufficient activation of CaN (Shi et al., 2000).

To address this possibility, we used a technique to activate CaN prematurely in P9 slices by stimulating the stratum opticum, which contains the afferent inputs to the SC (Shi et al., 2000). Stimulating these axons for $45 \mathrm{~min}$ in P9 WT SC slices induced a shortening of NMDAR currents in sSC neurons (Fig. $3 B)(p<0.05)$. On P9, the NMDAR current amplitude was not significantly different between NR2AKO and WT animals (Townsend et al., 2003). Therefore, we reasoned that electrical stimulation in mutant mice at this age should allow for a $\mathrm{Ca}^{2+}$ flux in NR2AKO neurons sufficient to permit the activation of $\mathrm{CaN}$. This procedure also avoids the potential confounds associ- ated with any developmental abnormalities in the retina that could arise from the absence of NR2A in these mutants. Nevertheless, unlike WT age-matched mice, $45 \mathrm{~min}$ of stimulation to the stratum opticum in the NR2AKO had no significant effect on the decay time of the NMDAR current (Fig. $3 B$ ) ( $p=0.49$ comparing before and after stimulation).

We also tested whether the persistence of long NMDAR currents in NR2AKO mice could be caused by abnormal CaN expression in these mutants. SDS PAGE was performed on whole lysate protein derived from the sSC of WT and NR2AKO mice. As shown in Figure 3C, no detectable differences in CaN levels between the two strains were present in the interval from P0 to P20.

\section{Constitutively active $\mathrm{CaN}$ has no effect on NMDAR current decay time in NR2AKO mice}

To test whether abnormal CaN activation or localization could be responsible for the lack of short NMDA currents in NR2AKO mice, we introduced a constitutively active $\mathrm{CaN}$ protein into the recording pipette solution. The decay time of the NMDAR current was assessed soon after breaking into the cell and again 40 min later after the $\mathrm{CaN}$ had sufficient time to diffuse out into the dendrites (Lledo et al., 1995). Figure 4, $A$ (top two traces) and $B$, shows that in P7 WT neurons, which contain mostly NR2B subunits (Shi et al., 2000), the decay time of NMDAR currents was only slightly faster $40 \mathrm{~min}$ after introducing constitutively active CaN. However, by P9-P10, constitutively active CaN reduced the NMDAR current $35 \pm 2.9 \%(p<0.05)$ (Fig. $4 A$, middle two traces, inset) after $40 \mathrm{~min}$ of dialyzing WT neurons. This change was not seen in P9-P10 WT neurons after constitutively active $\mathrm{CaN}$ was denatured, by boiling, before inclusion in the recording pipette. In contrast to WT neurons, sSC neurons in slices from NR2AKO mice showed no significant change in NMDAR current decay time using the constitutively active $\mathrm{CaN}$ (Fig. $4 A$, bottom two traces, $B$ ). These results indicate that $\mathrm{CaN}$ requires the NR2A subunit in the receptor to exert its developmental effect of shortening the decay time of NMDAR currents.

\section{NR2A dephosphorylation at Ser900 coincides with CaN- mediated effects on decay time}

Previous studies have shown that PKA and CaN may act directly on the NMDAR (Leonard and Hell, 1997; Krupp et al., 2002). The 


\section{A P7 WT NMDAR current}
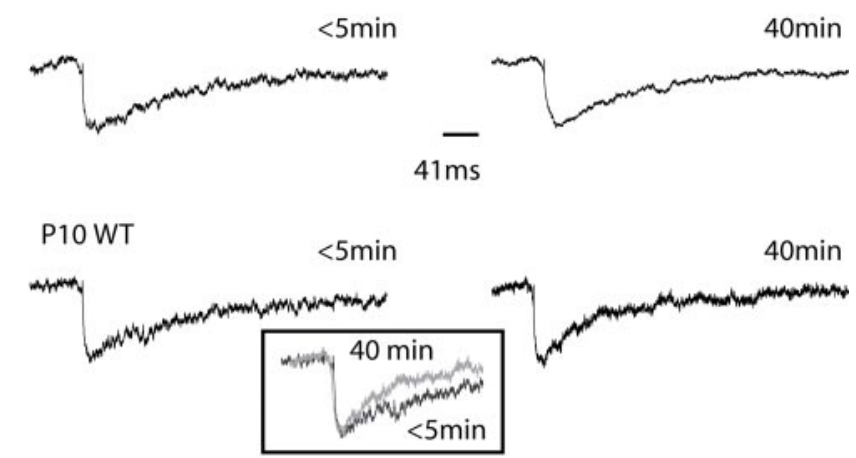

P10 NR2AKO
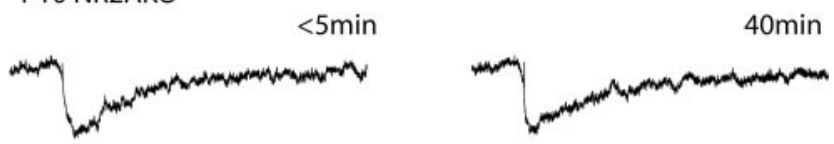

B Effect of Constitutively Active $\mathrm{CaN}$ on NMDAR current decay-time

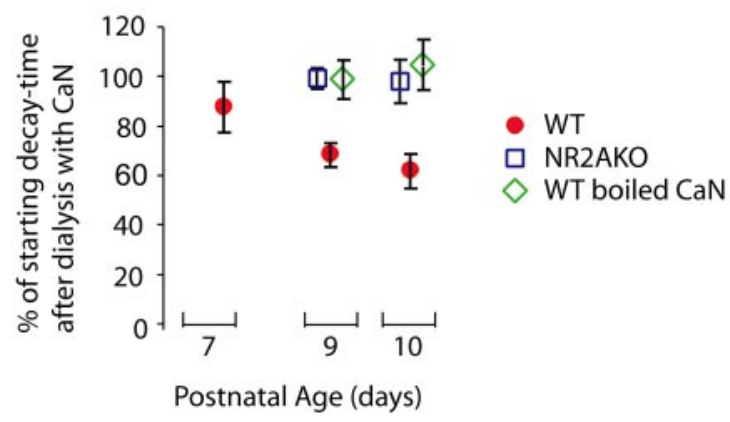

Figure 4. Constitutively active CaN shortens the decay time of NMDAR currents in WT P9P10 sSC neurons but not in age-matched NR2AK0 neurons. $A$, Infusion of a $1 \mu \mathrm{m}$ concentration of constitutively active $\mathrm{CaN} A \alpha$ subunit into sSCWT neurons through a whole-cell patch pipette had no effect on spontaneous NMDAR current decay time on P7. The same treatment shortened the NMDAR current decay time in P9 and P10 WT neurons at 40 min after the onset of infusion. (Inset shows overlay of $\angle 5$ and 40 min.) In contrast, P9 and P10 NR2AKO neurons failed to show changes in NMDAR current kinetics when tested in the same paradigm. B, Quantification of the percentage change in NMDAR current decay time between 5 and 40 min of dialyzing the cytoplasm of WT or NR2AKO neurons with constitutively active or heat-inactivated CaN. Both the heat-inactivated CaN in WT neurons and the constitutively active CaN in NR2AKO neurons failed to produce the age-dependent shortening in NMDAR current decay time (ANOVA TukeyKramer post hoc; $p<0.05$ for $\mathrm{P} 9$ and $\mathrm{P} 10 ; n \geq 4$ neurons per condition).

CaN-mediated dephosphorylation of NR2A produces a glycineindependent desensitization of the receptor. Such desensitization, if prolonged, could constitute the mechanism underlying the developmental downregulation of NMDAR decay time in vivo. Krupp et al. (2002) recently identified Ser900 on the NR2A subunit as a phosphorylation site regulated by $\mathrm{CaN}$. This site, when mutated to alanine, caused a persistent glycineindependent desensitization that was normally only seen after agonist was repetitively applied to human embryonic kidney 293 cells transfected with NR1 and the NR2A containing Ser900. Therefore, we developed a phospho-specific antibody directed against Ser900 on the NR2A subunit (anti-P-Ser900) to test whether the phosphorylation state of this residue was correlated with the developmental change in NMDAR current decay time in the sSC.
The anti-P-Ser900 antibody was characterized using dot blots with synthesized peptides spanning the Ser900 site. As shown in Figure 5A, the antibody has a much higher affinity for the phosphorylated peptide than the dephosphorylated peptide, confirming the affinity purification. In Western blots of synaptosomes derived from WT and NR2AKO mouse P9 sSC (an age when NMDARs are predicted to be phosphorylated based on the data shown in Fig. $1 A$ ), preimmune serum showed no affinity for NR2A. However, when the blot was stripped and reprobed using anti-P-Ser900, a band near $180 \mathrm{kDa}$ was detected in the lane containing WT but not NR2A KO protein (Fig. $5 B$ ). The additional band that appears at $\sim 90 \mathrm{kDa}$ is likely to be nonspecific, because it is pronounced in both the WT and NR2AKO lanes, and because the Ser900 is on the C terminus of NR2A, which is untranslated in the NR2AKO (Sakimura et al., 1995). Probing the same blot with anti-actin antibodies confirmed equal loading in both lanes.

If $\mathrm{CaN}$ modulates NMDAR current decay time through Ser900 of NR2A, then the phosphorylation of this residue should be reduced by P11 in WT mice when NMDAR currents show fast decay times. Western blotting was performed on synaptosomes collected from P9, P11, and P15 WT sSC and probed with antiP-Ser900. Figure $5 C$ shows that with equal loading of NR2A, only the lane containing P9 WT tissue showed significant reactivity to the P-Ser900 antibody. Optical density measurements were performed on samples run in triplicate. These are quantified in Figure $5 D$. The data demonstrate that the phosphorylation of Ser900 of NR2A is significantly reduced by P11, concurrent with CaNdependent shortening of NMDAR current decay time.

\section{NR2A phosphorylation at Ser900 is regulated by retinal activity}

If the phosphorylation state of Ser900 is correlated with NMDAR current decay time, then reducing retinal activity from P6 (as in Fig. 1) should maintain NR2A Ser900 in a phosphorylated state at P11. To test this, antag/Elvax was placed in one eye of WT mice on P6, and synaptosomes were prepared from the contralateral sSC on P11, whereas synaptosomes from the ipsilateral sSC served as a negative control. An additional control of sham/Elvax was also performed. Consistent with our hypothesis, Ser900 was significantly more phosphorylated in the SSC on P11 when retinal activity had been reduced from P6 than in controls (Fig. 6A, B). These data suggest that the phosphorylation state of Ser900 is highly correlated with the decay time of the NMDAR current. Thus, chronic dephosphorylation of this site on NR2A may account for the rapid onset, prolonged developmental shortening of NMDAR current decay time.

\section{Discussion}

Our previous work has shown that in the postnatal superior colliculus of rats, the decay time of the NMDAR current is shortened by $40-50 \%$ over a $24 \mathrm{hr}$ period preceding eye opening (Shi et al., 2000). We identified the phosphatase CaN in the postsynaptic neuron as involved in this shortening of NMDAR current decay time and demonstrated that the $\mathrm{Ca}^{2+}$ current mediated by the NMDAR was responsible for activating this phosphatase (Shi et al., 2000). Thus, during visual development in vivo, there is a posttranslational, activity-dependent, feedback mechanism that can quickly shorten the decay time of NMDAR currents.

The present studies reveal that, as in the rat, essentially all recorded neurons in the sSC of WT mice undergo this same developmentally regulated shortening of NMDAR current decay time on P10-P11. Acute application of the cell-permeable CaN 
inhibitor FK506, but not PP1/PP2A inhibitors, prolonged the NMDAR current decay time in P11 neurons, suggesting that CaN is likely to act directly on NMDARs or on some component of the NMDAR complex, as has been observed in excised patches (Lieberman and Mody, 1994) rather than through the PP1 pathway (Kato, 2000).

We also show that PKA counters the effect of CaN on NMDAR currents. Activation of PKA prolonged NMDAR current decay times, increasing NMDAR $\mathrm{Ca}^{2+}$ influx. Thus, before activation of $\mathrm{CaN}$ on P10, PKA helps to maintain NMDAR currents with slow kinetics. The preponderance of NR2B at these young synapses is also a likely factor in maintaining NMDAR currents with long decay times (as seen in Fig. 4A).

It was somewhat unexpected that increased retinal activity should bias the balance between PKA and CaN in favor of the phosphatase. In hippocampal models of synaptic plasticity, it has been found that low levels of activity favor phosphatase activation and depression of synapses, whereas higher levels of activity favor kinase activity and potentiation of synapses (Winder and Sweatt, 2001; Hedou and Mansuy, 2003). However, the stimulus used in Figure $3 B$ to activate $\mathrm{CaN}$ in the sSC is similar to a long-term depression protocol. Therefore, the elevated levels of activity in the sSC on P10 may more closely resemble low-frequency stimuli (Lo et al., 2002).

Because NR2B-containing NMDARs have long decay times, we anticipated that CaN might shorten the kinetics of NMDAR currents in mice that only express NR2B (NR2AKO mice). However, the current data show no significant developmental change in decay time of NMDAR currents in the NR2AKO mutants. CaN appears to be expressed normally in the sSC of these mutants, and constitutively active $\mathrm{CaN}$ infused in the postsynaptic neuron failed to shorten the decay time of NMDAR currents in mutant mice, although this treatment was consistently effective at decreasing the NMDAR current decay time prematurely in WT neurons of the same age (Fig. 4). Based on our observations, we conclude that CaN must act through the NR2A subunit to affect NMDAR function. This interpretation is consistent with a previous report suggesting that NR2B-containing receptors are less susceptible to glycine-independent desensitization (Krupp et al., 1996).

To date, the CaN effect on NMDAR kinetics in the developing sSC most closely resembles a form of glycine-independent desensitization, which reduces successive NMDAR currents evoked in cultured hippocampal neurons (Tong et al., 1995). This desensitization has been studied extensively and is known to involve allosteric changes in the extracellular domain of the NR2A sub-
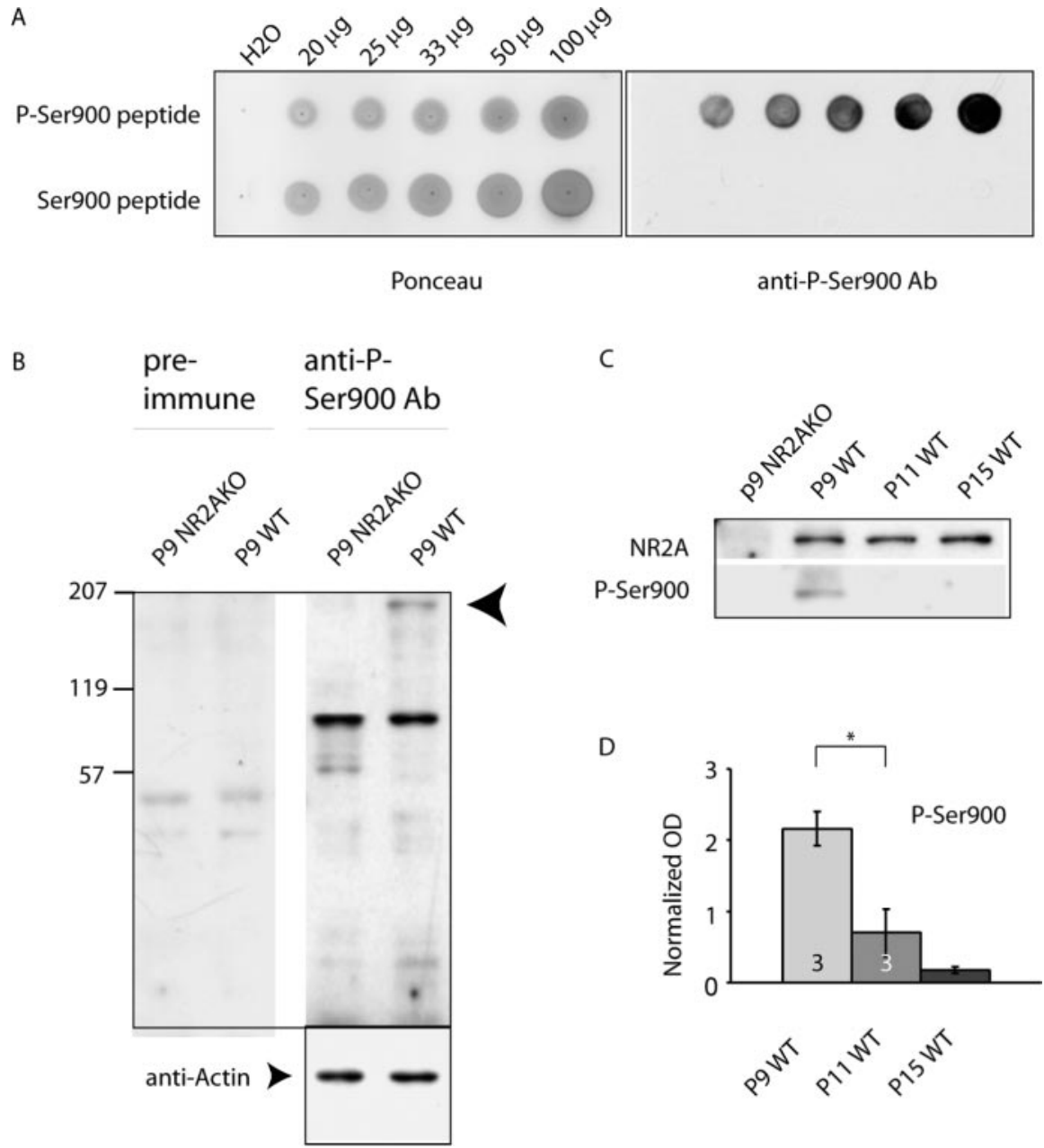

C

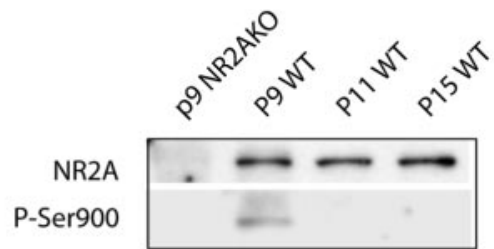

D
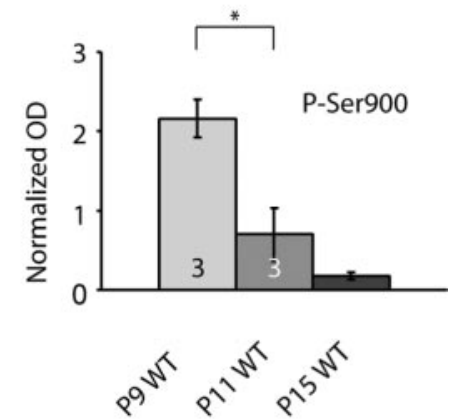

Figure 5. Dephosphorylation of Ser900 on NR2A in SSC neurons is detected with a phospho-specific antibody. A, A serial dilution (20-100 $\mu \mathrm{g}$ ) of the synthetic peptide (amino acids 895-906 of NR2A mouse sequence) spanning Ser900 was spotted was not. The same blot was stained with Ponceau (left) and then with anti-P-Ser900 antibody (right). B, Synaptosomes prepared 列

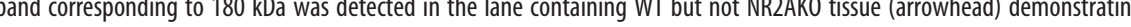
specific binding to NR2A and no cross-reactivity with NR2B. A nonspecific band appeared at $\sim 90 \mathrm{kDa}$ in both WT and NR2A (when NMDAR currents have long phosphorylation of Ser900 was significantly greater in P9 than in P11 or P15 WT synaptosomes (Student's $t$ test; ${ }^{*} p<0.05$ ). Phospho-NR2A was normalized against NR2A after stripping and reprobing the membrane (see Materials and Methods).

unit near the agonist binding site (Krupp et al., 1998; Villarroel et al., 1998). However, recent evidence has implicated phosphorylation of the cytoplasmic $\mathrm{C}$ terminal of the NR2A subunit in this desensitization. Ser900 of NR2A was of particular interest, because it can be dephosphorylated by $\mathrm{CaN}$, and mutation to alanine induces a persistently desensitized state (Krupp et al., 2002). Desensitization could be consistent with the faster NMDAR current decay time seen in the sSC after CaN activation. Consequently, we generated a phospho-specific antibody to the Ser900 site, and the present results demonstrate that the phosphorylation state of Ser900 is tightly correlated with the developmental profile of NMDAR current decay time in the sSC. Therefore, we propose that dephosphorylation of this site is responsible for mediating the CaN-dependent shortening of synaptic NMDAR currents during visual development. The NR2B amino acid sequence also contains a potential threonine phosphorylation site 


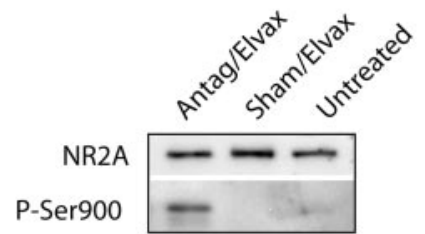

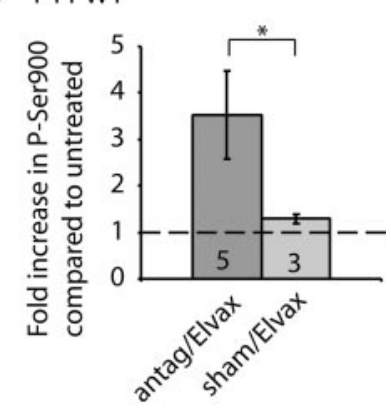

Figure 6. Dephosphorylation of Ser900 in $\mathrm{SSC}$ neurons is disrupted when visual activity is impaired by intraocular glutamate antagonists. A, Addition of glutamate receptor antagonists to one eye on P6 (antag/Elvax; as in Fig. 1C) prevented the dephosphorylation of NR2A in the contralateral $\mathrm{SSC}$ at P11. Because visual impairment also reduces levels of NR2A in synaptic fractions (Townsend et al., 2003), lanes were loaded for equal expression of NR2A. Sham/Elvaxtreated animals showed little or no P-Ser900 in the contralateral SSC at P11, similar to that seen in untreated littermates. B, Quantification of phospho-Ser900 demonstrated that visual impairment with intraocular antag/Elvax significantly increased phosphorylation of this site compared with sham or untreated animals shown as a dotted line (Student's $t$ test; ${ }^{*} p<0.05$ ). $n$ indicates the number of litters operated.

for PKA and CaN. However, our evidence with constitutively active $\mathrm{CaN}$ in the NR2AKO mouse suggests that any dephosphorylation of NR2B by CaN that might occur is not sufficient to shorten the decay time of the NMDAR.

If, as we suggest, the progressive loss of NR2A phosphorylation at Ser900 accounts for the rapid shortening of the NMDAR current decay time during this stage of development, then the addition of small amounts of phospho-NR2A to synapses may not be sufficient to induce shortened synaptic currents. Because, it is well established that NR1a/NR2A receptors are intrinsically faster than NR1a/NR2B receptors (Vicini et al., 1998), we suggest two hypotheses illustrated in Figure 7 to account for these data. During this period of synaptogenesis when NR2A-enriched receptors replace NR2B-enriched receptors (Tovar and Westbrook, 1999), some receptors may assemble containing both an NR2B and an NR2A subunit (Sheng et al., 1994; Luo et al., 1997). In model A, single NMDARs containing NR2A/NR2B subunits may show a distinctive decay time depending on the phosphorylation state of the NR2A subunit. In this scenario, a single receptor could transition between slow and fast decay times. Alternatively, in model B, a synapse contains a mix of NR2B-only and NR2A-only receptors. During the postsynaptic depolarization, the initial phase of the synaptic NMDAR current may show a strong dependence on the NR2A component (particularly because decay time is measured $90-37 \%$ peak amplitude) depending on the phosphorylation state of the NR2A-only receptors. The slow tail current, which falls below $37 \%$, would then be dominated by the NR2B-only receptors. Neither of these models is exclusive of the other, and both indicate that the decay time of NR2Acontaining receptors may be modulated by phosphorylation.

It will be important to determine the interval during development that $\mathrm{CaN}$ is responsible for maintaining short decay times. Our unpublished observations suggest that by P15, inhibitors of $\mathrm{CaN}$ have only a small effect on NMDAR currents. At this age, NMDAR currents already have fast decay times as a result of the eye-opening initiated addition of NR2A (Lu and ConstantinePaton, 2004). Presently, it is unclear why NMDARs at P15 are capable of maintaining fast currents in the presence of $\mathrm{CaN}$ inhibitors. Perhaps a second phosphorylation site (Ser929) on NR2A, identified by Krupp et al. (2002), becomes modified by

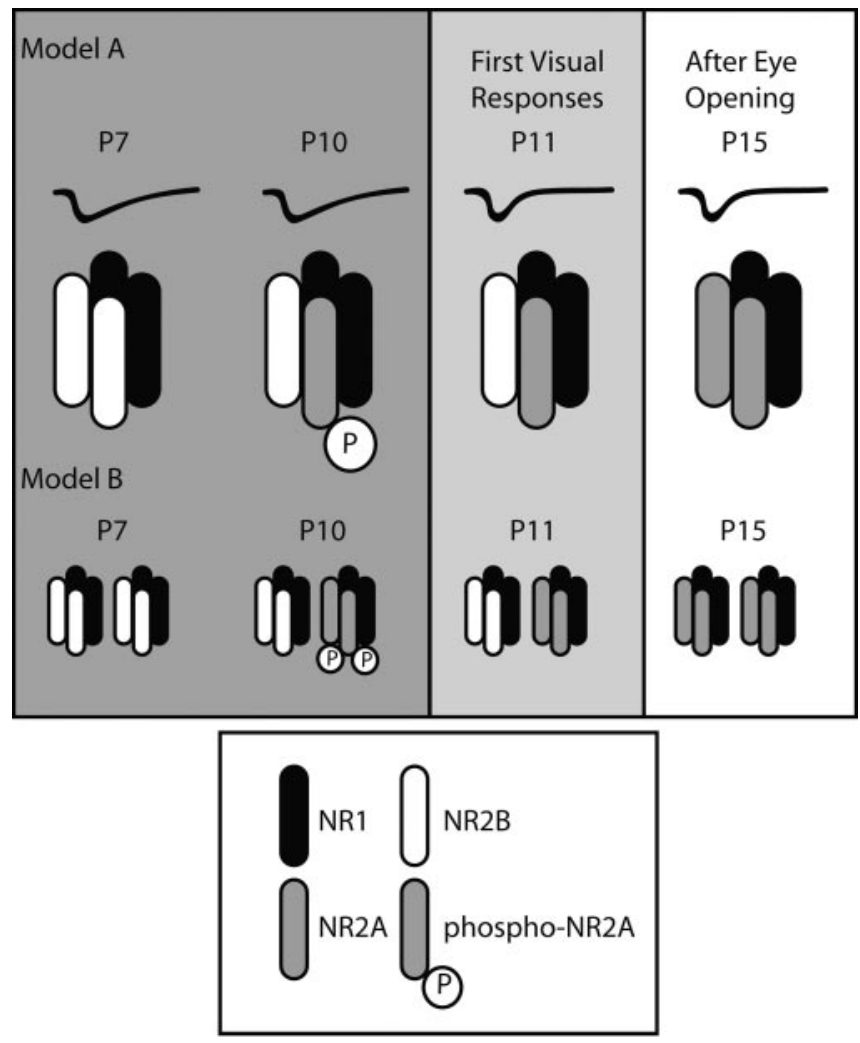

Figure 7. Two hypothetical models depicting how the phosphorylation of NR2A could rapidly modify the synaptic NMDAR current between P10 and P11. In both models, there is a gradual loss of NR2B-containing receptors between $\mathrm{P7}$ and $\mathrm{P} 15$ coinciding with the addition of NR2A-containing receptors. In model A, single NMDARs on P10-P11 contain both NR2A and NR2B subunits. The phosphorylation of NR2A in these receptors is regulated by PKA and CaN, depending on the amount of visual activity arriving from the retina. As NR2A becomes dephosphorylated, the function of these heteromeric receptors shifts in favor of shorter decay times by P11. In model B, synapses on P10 -P11 contain both NR2B-only and NR2A-only receptors, the latter of which is regulated by the phosphorylation-dephosphorylation cycle. Even if NR2Aonly receptors are intrinsically faster than NR2B-only receptors, the phosphorylation status of the NR2A-only receptors shortens the current decay time further. This additional shortening would be particularly pronounced in the initial phase of the synaptic NMDAR current, which is dominated by the faster response of the NR2A-containing receptors, whereas the slower NR2B component would predominate in the tail. Thus, between P10 and P11, there is extensive dephosphorylation of NR2A-only receptors, which causes a shift in the decay time of the synaptic current. In neither model is it necessary that all NR2A subunits become dephosphorylated; rather, as Figure 5D suggests, there is likely to be a continuous loss of phospho-NR2A until P15.

P15 and protects against phosphorylation of Ser900. Alternatively, visual activity may slowly inactivate PKA. For example, CaN has been shown to decrease CAMP by inactivating adenylate cyclase VIII in forebrain (Antoni et al., 1998). Finally, NR2A subunits may indeed have intrinsically faster kinetics than NR2B subunits, yet the loss of NR2B by $\mathrm{P} 15$ precludes the re-emergence of slow NMDAR currents for the reasons described above and in Figure 7. Thus, the effect of CaN on NMDAR decay time may be most robust only early in the second postnatal week when many sSC synapses contain receptors consisting of both NR2A and NR2B subunits and NMDAR $\mathrm{Ca}^{2+}$ influx is prolonged.

Our results also reveal that retinal activity can affect synapses in the CNS before eye opening. It has been recently demonstrated that the lateral geniculate neuron receptive fields show significant refinement in the ferret before eye opening (Akerman et al., 2004). Furthermore, by P10 in mice, photoreceptor activity begins to drive retinal ganglion cell activity (Tian and Copenhagen, $2001,2003)$. Similarly in rats, photic responses are detected in the 
SC early in the second postnatal week (Molotchnikoff and Itaya, 1993). For these reasons, we predicted that early light-driven activity in the retina may begin to drive visual neurons of the sSC and initiate the CaN-mediated change. Previous investigations have shown that the photoreceptor-bipolar-ganglion cell pathway is glutamatergic (Itaya et al., 1995; Bansal et al., 2000; Wong et al., 2000). Our data indicate that this pathway can be impaired in the early retina by infiltrating one eye cup with AMPA/KAR and NMDAR antagonists. Invariably, we observed the persistence of long NMDAR currents even by P11 and phospho-Ser900 in the sSC contralateral to eyes treated with antag/Elvax. Thus, these data provide direct evidence that visually driven synaptic responses in CNS neurons are altered significantly by light before eye opening initiates high-contrast patterned vision.

In conclusion, we have demonstrated in mice that NMDAR current decay time is rapidly modified by $\mathrm{CaN}$ at the close of retinocollicular map refinement. The importance of the NR2A subunit in this posttranslational regulation of the NMDAR current indicates a significant interaction between kinase-phosphatase cascades and insertion of new NR2A-containing NMDARs. $\mathrm{CaN}$ is poised to rapidly respond to changes in calcium flux through the NMDAR and mediate a negative feedback mechanism that rapidly downregulates the NMDAR current in response to visual activity. The slower substitution of NR2Aenriched NMDARs should maintain fast NMDAR kinetics even in the absence of $\mathrm{CaN}$, as long as NR2A receptor subunits predominate in the active receptors.

\section{References}

Akerman CJ, Grubb MS, Thompson ID, Tolhurst DJ, Morgan JE, Baker GE, Smyth D, Krug K (2004) Relay of visual information to the lateral geniculate nucleus and the visual cortex in albino ferrets. J Neurosci 24:170-182.

Ali DW, Salter MW (2001) NMDA receptor regulation by Src kinase signalling in excitatory synaptic transmission and plasticity. Curr Opin Neurobiol 11:336-342.

Antoni FA, Palkovits M, Simpson J, Smith SM, Leitch AL, Rosie R, Fink G, Paterson JM (1998) $\mathrm{Ca}^{2+} /$ calcineurin-inhibited adenylyl cyclase, highly abundant in forebrain regions, is important for learning and memory. J Neurosci 18:9650-9661.

Bansal A, Singer JH, Hwang BJ, Xu W, Beaudet A, Feller MB (2000) Mice lacking specific nicotinic acetylcholine receptor subunits exhibit dramatically altered spontaneous activity patterns and reveal a limited role for retinal waves in forming ON and OFF circuits in the inner retina. J Neurosci 20:7672-7681.

Carmignoto G, Vicini S (1992) Activity-dependent decrease in NMDA receptor responses during development of the visual cortex. Science 258:1007-1011.

Colledge M, Dean RA, Scott GK, Langeberg LK, Huganir RL, Scott JD (2000) Targeting of PKA to glutamate receptors through a MAGUK-AKAP complex. Neuron 27:107-119.

Dunah AW, Standaert DG (2001) Dopamine D1 receptor-dependent trafficking of striatal NMDA glutamate receptors to the postsynaptic membrane. J Neurosci 21:5546-5558.

Hedou G, Mansuy IM (2003) Inducible molecular switches for the study of long-term potentiation. Philos Trans R Soc Lond B Biol Sci 358:797-804.

Itaya SK, Fortin S, Molotchnikoff S (1995) Evolution of spontaneous activity in the developing rat superior colliculus. Can J Physiol Pharmacol 73:1372-1377.

Kato K (2000) The role of calcineurin on the induction of synaptic plasticity. Nihon Shinkei Seishin Yakurigaku Zasshi 20:189-198.

Krupp JJ, Vissel B, Heinemann SF, Westbrook GL (1996) Calciumdependent inactivation of recombinant $N$-methyl-D-aspartate receptors is NR2 subunit specific. Mol Pharmacol 50:1680-1688.

Krupp JJ, Vissel B, Heinemann SF, Westbrook GL (1998) N-terminal domains in the NR2 subunit control desensitization of NMDA receptors. Neuron 20:317-327.

Krupp JJ, Vissel B, Thomas CG, Heinemann SF, Westbrook GL (2002) Cal- cineurin acts via the C-terminus of NR2A to modulate desensitization of NMDA receptors. Neuropharmacology 42:593-602.

Leonard AS, Hell JW (1997) Cyclic AMP-dependent protein kinase and protein kinase $\mathrm{C}$ phosphorylate $\mathrm{N}$-methyl-D-aspartate receptors at different sites. J Biol Chem 272:12107-12115.

Lieberman DN, Mody I (1994) Regulation of NMDA channel function by endogenous $\mathrm{Ca}(2+)$-dependent phosphatase. Nature 369:235-239.

Lindlbauer R, Mohrmann R, Hatt H, Gottmann K (1998) Regulation of kinetic and pharmacological properties of synaptic NMDA receptors depends on presynaptic exocytosis in rat hippocampal neurones. J Physiol (Lond) 508:495-502.

Lledo PM, Hjelmstad GO, Mukherji S, Soderling TR, Malenka RC, Nicoll RA (1995) Calcium/calmodulin-dependent kinase II and long-term potentiation enhance synaptic transmission by the same mechanism. Proc Natl Acad Sci USA 92:11175-11179.

Lo FS, Mize RR, Cork RJ, Namkung Y, Shin HS (2002) Properties of LTD and LTP of retinocollicular synaptic transmission in the developing rat superior colliculus. Eur J Neurosci 15:1421-1432.

Lu W, Constantine-Paton M (2004) Eye opening rapidly induces synaptic potentiation and refinement. Neuron 43:237-249.

Luo J, Wang Y, Yasuda RP, Dunah AW, Wolfe BB (1997) The majority of $\mathrm{N}$-methyl-D-aspartate receptor complexes in adult rat cerebral cortex contain at least three different subunits (NR1/NR2A/NR2B). Mol Pharmacol 51:79-86.

Malleret G, Haditsch U, Genoux D, Jones MW, Bliss TV, Vanhoose AM, Weitlauf C, Kandel ER, Winder DG, Mansuy IM (2001) Inducible and reversible enhancement of learning, memory, and long-term potentiation by genetic inhibition of calcineurin. Cell 104:675-686.

McLaughlin T, Torborg CL, Feller MB, O'Leary DD (2003) Retinotopic map refinement requires spontaneous retinal waves during a brief critical period of development. Neuron 40:1147-1160.

Miller ED, Tran MN, Wong GK, Oakley DM, Wong RO (1999) Morphological differentiation of bipolar cells in the ferret retina. Vis Neurosci 16:1133-1144.

Molotchnikoff S, Itaya SK (1993) Functional development of the neonatal rat retinotectal pathway. Brain Res Dev Brain Res 72:300-304.

Monyer H, Burnashev N, Laurie DJ, Sakmann B, Seeburg PH (1994) Developmental and regional expression in the rat brain and functional properties of four NMDA receptors. Neuron 12:529-540.

Nase G, Weishaupt J, Stern P, Singer W, Monyer H (1999) Genetic and epigenetic regulation of NMDA receptor expression in the rat visual cortex. Eur J Neurosci 11:4320-4326.

Perrino BA, Fong YL, Brickey DA, Saitoh Y, Ushio Y, Fukunaga K, Miyamoto E, Soderling TR (1992) Characterization of the phosphatase activity of a baculovirus-expressed calcineurin A isoform. J Biol Chem 267:15965-15969.

Prusky GT, Ramoa AS (1999) Novel method of chronically blocking retinal activity. J Neurosci Methods 87:105-110.

Quinlan EM, Olstein DH, Bear MF (1999) Bidirectional, experiencedependent regulation of $\mathrm{N}$-methyl-D-aspartate receptor subunit composition in the rat visual cortex during postnatal development. Proc Natl Acad Sci USA 96:12876-12880.

Raman IM, Tong G, Jahr CE (1996) Beta-adrenergic regulation of synaptic NMDA receptors by cAMP-dependent protein kinase. Neuron 16:415-421.

Rosenmund C, Stern-Bach Y, Stevens CF (1998) The tetrameric structure of a glutamate receptor channel. Science 280:1596-1599.

Rostas JA, Brent VA, Voss K, Errington ML, Bliss TV, Gurd JW (1996) Enhanced tyrosine phosphorylation of the $2 \mathrm{~B}$ subunit of the $\mathrm{N}$-methyl-Daspartate receptor in long-term potentiation. Proc Natl Acad Sci USA 93:10452-10456.

Sakimura K, Kutsuwada T, Ito I, Manabe T, Takayama C, Kushiya E, Yagi T, Aizawa S, Inoue Y, Sugiyama H, Mishina M (1995) Reduced hippocampal LTP and spatial learning in mice lacking NMDA receptor $\epsilon 1$ subunit. Nature 373:151-155.

Sheng M, Cummings J, Roldan LA, Jan YN, Jan LY (1994) Changing subunit composition of heteromeric NMDA receptors during development of rat cortex. Nature 368:144-147.

Shi J, Aamodt SM, Constantine-Paton M (1997) Temporal correlations between functional and molecular changes in NMDA receptors and GABA neurotransmission in the superior colliculus. J Neurosci 17:6264-6276. 
Shi J, Townsend M, Constantine-Paton M (2000) Activity-dependent induction of tonic calcineurin activity mediates a rapid developmental downregulation of NMDA receptor currents. Neuron 28:103-114.

Simon DK, Prusky GT, O'Leary DD, Constantine-Paton M (1992) $\mathrm{N}$-methyl-D-aspartate receptor antagonists disrupt the formation of a mammalian neural map. Proc Natl Acad Sci USA 89:10593-10597.

Tian N, Copenhagen DR (2001) Visual deprivation alters development of synaptic function in inner retina after eye opening. Neuron 32:439-449.

Tian N, Copenhagen DR (2003) Visual stimulation is required for refinement of ON and OFF pathways in postnatal retina. Neuron 39:85-96.

Tong G, Shepherd D, Jahr CE (1995) Synaptic desensitization of NMDA receptors by calcineurin. Science 267:1510-1512.

Tovar KR, Westbrook GL (1999) The incorporation of NMDA receptors with a distinct subunit composition at nascent hippocampal synapses in vitro. J Neurosci 19:4180-4188.

Townsend M, Yoshii A, Mishina M, Constantine-Paton M (2003) Developmental loss of miniature $N$-methyl-D-aspartate receptor currents in NR2A knock-out mice. Proc Natl Acad Sci USA 100:1340-1345.

Vicini S, Wang JF, Li JH, Zhu WJ, Wang YH, Luo JH, Wolfe BB, Grayson DR (1998) Functional and pharmacological differences between recombinant $N$-methyl-D-aspartate receptors. J Neurophysiol 79:555-566.

Villarroel A, Regalado MP, Lerma J (1998) Glycine-independent NMDA receptor desensitization: localization of structural determinants. Neuron 20:329-339.

Wang LY, Orser BA, Brautigan DL, MacDonald JF (1994a) Regulation of NMDA receptors in cultured hippocampal neurons by protein phosphatases 1 and 2A. Nature 369:230-232.

Wang LY, Dudek EM, Browning MD, MacDonald JF (1994b) Modulation of AMPA/kainate receptors in cultured murine hippocampal neurones by protein kinase C. J Physiol (Lond) 475:431-437.

Watanabe M, Inoue Y, Sakimura K, Mishina M (1992) Developmental changes in distribution of NMDA receptor channel subunit mRNAs. NeuroReport 3:1138-1140.

Winder DG, Sweatt JD (2001) Roles of serine/threonine phosphatases in hippocampal synaptic. Nat Rev Neurosci 2:461-474.

Wong WT, Myhr KL, Miller ED, Wong RO (2000) Developmental changes in the neurotransmitter regulation of correlated spontaneous retinal activity. J Neurosci 20:351-360.

Zeng H, Chattarji S, Barbarosie M, Rondi-Reig L, Philpot BD, Miyakawa T, Bear MF, Tonegawa S (2001) Forebrain-specific calcineurin knock-out selectively impairs bidirectional synaptic plasticity and working/episodiclike memory. Cell 107:617-629.

Zhuo M, Zhang W, Son H, Mansuy I, Sobel RA, Seidman J, Kandel ER (1999) A selective role of calcineurin alpha in synaptic depotentiation in hippocampus. Proc Natl Acad Sci USA 96:4650-4655. 